'Departamento de Salud Mental y Psiquiatría, Facultad de Medicina, Universidad de La Frontera. Temuco, Chile.

2Departamento de Psiquiatría, Facultad de Medicina, Universidad de Valparaíso. Valparaíso, Chile.

${ }^{3}$ Departamento de Psiquiatría, Facultad de Medicina, Pontificia Universidad

Católica de Chile. Santiago, Chile. ${ }^{4}$ Departamento de Psicología, Facultad de Ciencias Sociales, Universidad de La Frontera. Temuco, Chile.

${ }^{5}$ Department of Psychology, University of California-Los Angeles. Los Ángeles, Estados Unidos.

${ }^{6}$ Escuela de Salud Pública, Facultad de Medicina, Universidad de Chile, Santiago, Chile

Centro de Salud Mental y Psiquiatría Ambulatoria, Hospital del Salvador, Valparaíso, Chile.

apsicólogo; Doctor en Psicobiología.

bsicólogo; Doctor en Psicología de la Salud.

‘Kinesióloga, Estudiante de Magíster en Epidemiología Clínica. ¿Psicóloga Clínica.

Trabajo financiado por Proyecto FONIS SA09120020.

Recibido el 4 de junio de 2012, aceptado el 19 de julio de 2012.

Correspondencia a: Dr. Jaime R. Silva, PhD. Departamento de Salud Mental y Psiquiatría, Facultad de Medicina, Universidad de La Frontera. Av. Alemania 0458, Temuco. Fono: 45-592177 E-mail: jaimesilva@ufro.cl

\section{Estudio multicéntrico para la validación de la versión en español del Eating Disorder Diagnostic Scale}

\author{
JAIME R. SILVA ${ }^{1, a}$, ROSA BEHAR ${ }^{2}$, PATRICIA CORDELLA ${ }^{3}$, \\ MANUEL ORTIZ ${ }^{4,5, b}$, KARINA JARAMILLO ${ }^{1, \mathrm{c}}$, \\ RUBÉN ALVARADO ${ }^{6}$, MARÍA JOSÉ JORQUERA $^{7, d}$
}

\section{Validation of the Spanish version of the Eating Disorders Diagnostic Scale}

\begin{abstract}
Background: The Eating Disorders Diagnostic Scale (EDDS) is a self-administered low cost psychometric instrument with excellent levels of temporal reliability and validity. Aim: To adapt and validate the EDDS in Chile. Material and Methods: The factorial structure, internal consistency and test-retest reliability of the Spanish-language version of the EDDS was analyzed in a sample of 1964 university and high school students. The concurrent validity was tested in a sample of 50 primary care patients with ED and 59 controls, comparing its results with those of a structured psychiatric interview (CIDI). Results: The EDDS showed a high internal consistency, moderate test-retest reliability, an appropriate factorial structure (in women) and an excellent convergent validity. Also, the diagnosis of ED obtained with the EDDS is moderately consistent with the structured psychiatric interview. Conclusions: The Spanish-language version of the EDDS showed a satisfactory psychometric behavior and a good capacity for detecting ED, according to the DSM criterion.
\end{abstract}

(Rev Med Chile 2012; 140: 1562-1570).

Key words: Eating disorders; Diagnosis; Self report; Validation studies.
L os trastornos de la conducta alimentaria (TCA) son una constelación de enfermedades de importancia creciente para los sistemas de salud alrededor del mundo ${ }^{1-6}$. Dentro de la heterogénea diversidad de manifestaciones clínicas de los TCA se han destacado tradicionalmente dos; la anorexia y la bulimia nerviosa ${ }^{7}$. Actualmente, otras presentaciones también han cobrado vigor, especialmente el trastorno por atracón alimentario $^{8-10}$. Estudios epidemiológicos internacionales muestran que los TCA se presentan en el rango de $3 \%$ a $10 \%$ de la población de riesgo, es decir, de mujeres entre 15 y 29 años $^{11-13}$.

Considerando el ámbito clínico y de investigación de los TCA, en Chile es posible describir al menos dos tipos de problemáticas. En primer lugar, salvo reportes anecdóticos y un estudio preliminar $^{14}$, se desconoce la real prevalencia de los TCA. Considerando los niveles preventivo y de formulación de políticas públicas, lo que es una carencia relevante. La segunda, es que la mayoría de las personas con TCA no reciben tratamiento. Uno de los factores determinantes de esto es la inadecuada pesquisa de la enfermedad ${ }^{15,16}$. Por ejemplo, se ha reportado que en los servicios de atención primaria alrededor de $40 \%$ de las personas con TCA no son derivados a atención especializada y quedan sin tratamiento adecuado ${ }^{17}$.

Un paso no trivial para superar la situación descrita es disponer de herramientas diagnósticas 
económicas, confiables y válidas. En la actualidad, algunos grupos de investigación y de atención clínica utilizan instrumentos que tienen un valor indudable, pero que no permiten obtener confiablemente un diagnóstico de TCA. Por ejemplo, el Test de Actitudes Alimentarias ${ }^{18}$, y el Inventario de Desórdenes Alimentarios (EDI) ${ }^{19}$ permiten evaluar diversos aspectos de la conducta alimentaria pero en ningún caso generan un diagnóstico confiable de TCA. Por ende, las condiciones actuales relacionadas a los instrumentos de evaluación diagnóstica de TCA necesariamente deben ser mejoradas.

Existe la posibilidad de enfrentar eficientemente este problema. Un equipo multidisciplinario de las Universidades de Stanford, de Washington y Texas, en Estados Unidos de Norteamérica, elaboraron la Escala de Diagnóstico de Desórdenes Alimentarios (Eating Disorder Diagnostic Scale; EDDS) ${ }^{20}$. El EDDS es capaz de entregar un diagnóstico clínicamente confiable por sí mismo, lo que lo hace un instrumento propicio para la investigación básica, la epidemiología, la evaluación de programas de prevención y tratamiento, y para los diversos setting clínicos donde se requiere el diagnóstico de estas enfermedades ${ }^{20,21}$. Efectivamente, el instrumento ha mostrado sensibilidad frente a programas de intervención ${ }^{21}$ y sus parámetros de confiabilidad y validez han resultado apropiados, incluso en estudios internacionales ${ }^{22,23}$.

Por todo lo anterior, se propuso hacer la adaptación y validación del EDDS al contexto nacional. Se realizó un estudio multicéntrico cuyo objetivo fundamental fue estimar las propiedades psicométricas de la versión en español del instrumento para su utilización en población chilena. Se plantearon cinco hipótesis; H1) El EDDS es un instrumento psicométrico confiable; H2) tendrá niveles apropiados de fiabilidad y fiabilidad test/ re-test; H3) presentará una estructura factorial similar a la versión original; H4) se asociará a niveles elevados de sintomatología alimentaria evaluada con el EDI-2 y; H5) el diagnóstico de TCA obtenido con el EDDS será concordante con el obtenido mediante el gold standard.

\section{Material y Método}

La investigación se trató de dos estudios secuenciales, respetando normas éticas concordantes con la Declaración de Helsinki (2008) y aprobados por el Comité de Ética del Servicio de Salud, Araucanía Sur.

\section{Estudio 1: Estructura factorial, consistencia interna, fiabilidad test-retest y validez de constructo de la versión en español del EDDS en una muestra de estudiantes universitarios y secundarios}

\section{Participantes}

Por medio de un muestreo no probabilístico intencionado, se seleccionó una muestra de 1.964 participantes de las ciudades de Temuco, Valparaíso, Viña del Mar y Santiago. Los criterios de inclusión fueron: a) personas mayores de 13 años; b) estudiantes secundarios y universitarios; c) pertenecientes a nivel socioeconómico alto, medio y bajo. Se utilizó un tamaño muestral de esta magnitud para garantizar un nivel aceptable de estabilidad de los factores del instrumento ${ }^{24}$.

\section{Instrumentos}

1) Escala de Diagnóstico de Desórdenes Alimentarios (EDDS) ${ }^{20 *}$. Esta escala de 22 ítems que evalúa tres categorías diagnósticas (anorexia nerviosa, bulimia nerviosa y trastorno por atracón), un diagnóstico "sub-umbral" (presencia de todos los síntomas del desorden pero al menos uno en una severidad sub-diagnóstica) y un nivel general de problemas alimentarios (compuesto sintomático del EDDS).

2) Inventario de Trastornos Alimentarios-2 (Eating Disorders Inventory-2) ${ }^{19}$. El EDI-2 consiste en 64 proposiciones distribuídas en 8 factores relativos a la alimentación y sus alteraciones. El un instrumento psicométrico tipo Likert y validado en Chile ${ }^{25}$.

3) Escala de Restricción (RRS) ${ }^{26,27}$. Para la medición de la variable restricción alimentaria ${ }^{28-30}$, se utilizó la versión en español validada en Chile ${ }^{31,32}$, la cual mide dos factores: preocupación por la dieta y fluctuaciones de peso.

\section{Procedimiento}

En el contexto de una aplicación colectiva, un asistente de investigación presentó el estudio como "una investigación para conocer la conducta y emociones en estudiantes" y leyó las instrucciones generales. Posteriormente, cada participante

\footnotetext{
* Para obtener el EDDS en español solicitar una copia al autor de correspondencia. También se encuentra disponible un software para la corrección informatizada del instrumento en: http://silvalab.neurociencia.cl/edds
} 
Validación de la versión en español del Eating Disorder Diagnostic Scale - J. R. Silva et al

recibió un cuadernillo de auto-reportes para los que disponían de 45 min para contestar. Cada individuo respondió anónimamente y tuvo libertad de abandonar el estudio en cualquier momento. Un subgrupo de participantes respondió los autoreportes por segunda vez en un rango de 30-45 días, siguiendo el mismo protocolo de aplicación.

\section{Estudio 2: Validez concurrente de la versión en español del EDDS con el CIDI 3.0}

\section{Participantes}

La población del estudio incluyó 50 pacientes usuarios de los centros de salud San Joaquín (PUC), Servicio Médico de Alumnos de la Universidad de Valparaíso y del Servicio de Psiquiatría del Hospital Hernán Henríquez de Temuco durante el año 2010. Los participantes seleccionados cumplían de los siguientes criterios: a) pacientes diagnosticados por un Psiquiatra; b) pacientes que poseen el diagnóstico de alguno de los TCA, c) sin comorbilidad psiquiátrica del eje I. El grupo control estuvo conformado por 59 sujetos encuestados en el estudio 1 (ver procedimiento a continuación).

\section{Instrumentos}

1) Escala de Diagnóstico de Desórdenes Alimentarios (EDDS) ${ }^{20}$. Ver Estudio 1.

2) Entrevista Psiquiátrica Estructurada $\mathrm{CIDI}^{33}$. El CIDI es una entrevista estructurada para la evaluación de desórdenes mentales de acuerdo a criterios del DSM-IV-TR ${ }^{34}$ y el ICD- $10^{35}$. Es administrada por un entrevistador entrenado y su diseño fue pensado para el uso en estudios epidemiológicos y clínicos. Se utilizó la versión 3.0, cuya encuesta está infomatizada.

\section{Procedimiento}

A partir de la información disponible de pacientes que se atendían en los centros reclutados, se seleccionó una muestra de 50 sujetos diagnosticados con algún TCA durante los últimos 3 meses previos. De los sujetos encuestados en el estudio 1, por medio de un procedimiento de emparejamiento, se procedió a la seleccionar 59 sujetos controles. Las variables de emparejamiento fueron a) sexo; b) edad; c) nivel socioeconómico. Posteriormente, los participantes fueron citados a sus respectivos centros de referencia, donde fueron entrevistados con el CIDI. Finalmente, respondieron la versión final del EDDS, obtenida del estudio 1. Todos los sujetos recibieron una indemnización por su participación.

\section{Resultados}

\section{Estudio 1}

La muestra total estuvo conformada por 1.964 estudiantes secundarios y universitarios. De este grupo 982 respondieron el cuestionario en dos ocasiones (Tabla 1). De acuerdo a la evaluación obtenida por el EDDS los TCA mostraron una alta frecuencia (Tabla 2).

\section{Consistencia interna}

$\mathrm{La}$ inspección de la confiabilidad interna muestra resultados satisfactorios. La escala total (considerando los ítems continuos) alcanza un alpha de Cronbach de 0,81 en mujeres y 0,79 en

\section{Tabla 1. Estadísticos descriptivos de muestra} total

\begin{tabular}{|lcrrc|}
\hline & Mujeres & Hombres & t & p \\
$\mathrm{n}$ & 1.218 & 749 & & \\
Edad & 17,64 & 16,84 & 5,668 & $0,000^{*}$ \\
IMC & 21,81 & 22,17 & $-2,136$ & $0,033^{*}$ \\
PD & 8,08 & 5,25 & 14,398 & $0,000^{*}$ \\
FP & 3,92 & 3,57 & 2,157 & $0,031^{*}$ \\
EddsCom & 21,24 & 12,86 & 11,425 & $0,000^{*}$ \\
\hline
\end{tabular}

${ }^{*} \mathrm{p}<0,05$; IMC = Índice de Masa Corporal, PD = Preocupación por la dieta, FP = Fluctuaciones de peso; EddsCom = Compuesto sintomático del EDDS.

Tabla 2. Porcentaje de diagnósticos según EDDS muestra total

\begin{tabular}{|lccc|}
\hline & Mujeres & Hombres & Total \% \\
\hline Normal & 82,2 & 93,2 & 86,4 \\
\hline Anorexia & 0,4 & 0 & 0,3 \\
\hline Bulimia & 6,1 & 3,3 & 5,1 \\
\hline TPA & 3,1 & 0,4 & 2,1 \\
\hline Anorexia sub & 2,7 & 0,4 & 1,8 \\
Bulimia sub & 4,9 & 2,6 & 4,0 \\
\hline TPA sub & 0,4 & 0,1 & 0,3 \\
\hline
\end{tabular}

Sub = Diagnóstico subumbral; TPA = Trastorno por atracón 
hombres. Considerando la muestra total el alpha de Cronbach fue de 0,84. En el caso de los hombres, el ítem 16 no resulta útil como medida psicométrica (índice de homogeneidad corregida $=0,254$ ).

\section{Confiabilidad test-restest}

En la Tabla 3 se presenta el grado de acuerdo entre la primera y segunda aplicación del EDDS según sexo. En el caso de las mujeres, el valor de Kappa del acuerdo entre las dos administraciones para las 7 categorías diagnósticas fue 0,41 y de 0,45 para la correspondencia entre "cualquier diagnóstico". Para los hombres los valores fueron 0,40 y 0,48 , respectivamente.

\section{Estructura factorial}

El análisis factorial exploratorio comenzó con la evaluación de su pertinencia. La prueba de KMO obtuvo un valor de 0,88 en mujeres y 0,82 en hombres, lo cual sugiere una excelente adecuación para la ejecución de un análisis factorial. Asimismo, la prueba de esfericidad de Bartlett devela que existen relaciones entre las variables que pueden ser analizadas (ambos $\mathrm{p}<0,000$ ).

En el caso de las mujeres el Análisis de Compo- nentes Principales extrajo 4 componentes lineales en los datos cuyos auto-valores fueron igual o mayor a 1, que explican un total de $62,13 \%$ de la varianza. La rotación oblicua también resultó en una estructura de cuatro factores (Tabla 4). Los cuatro componentes según Lee y colaboradores ${ }^{23}$ corresponden a un factor de insatisfacción corporal (ítems 1-4) de conductas de atracón (ítems 5-6 y 9-14), de frecuencia de atracón (ítems 7 y 8 ) y, finalmente, de frecuencia de conductas compensatorias (ítems 15-18). La inspección de la Tabla 4 muestra que el ítem 12 no carga adecuadamente en ningún factor.

En el caso de los hombres se extrajo 6 componentes lineales que explican el 70,96\% de la varianza. En este caso la estructura factorial mantiene el factor de insatisfacción corporal (ítems 1-4) y el de frecuencia de atracón (ítems 7 y 8 ).

\section{Validez convergente}

Para obtener indicadores de validez convergente se obtuvo las correlaciones entre el compuesto sintomático del EDDS y los factores relevantes de la escala EDI-2 y la RRS (Tabla 5). Asimismo, se

Tabla 3. Tabla de contingencias, según sexo, entre primera y segunda aplicación para (a) todos los diagnósticos y (b) presencia o ausencia de trastornos de la conducta alimentaria

\begin{tabular}{|c|c|c|c|c|c|c|c|c|c|c|c|c|c|c|c|c|}
\hline \multirow[b]{2}{*}{ Tiempo 2} & \multicolumn{8}{|c|}{ Mujeres } & \multicolumn{8}{|c|}{ Hombres } \\
\hline & 0 & 1 & 2 & 3 & 4 & 5 & 6 & Total & 0 & 1 & 2 & 3 & 4 & 5 & 6 & Total \\
\hline \multicolumn{17}{|l|}{ Tiempo 1} \\
\hline 0. Sin diagnóstico & 450 & 2 & 12 & 8 & 5 & 13 & 4 & 494 & 340 & 0 & 1 & 2 & 0 & 9 & 0 & 352 \\
\hline 1. Anorexia & 0 & 1 & 0 & 0 & 1 & 0 & 0 & 2 & 0 & 0 & 0 & 0 & 0 & 0 & 0 & 0 \\
\hline 2. Bulimia & 18 & 0 & 13 & 1 & 0 & 4 & 0 & 36 & 5 & 0 & 4 & 1 & 0 & 2 & 0 & 12 \\
\hline 3. TPA & 8 & 0 & 1 & 10 & 0 & 0 & 1 & 20 & 0 & 0 & 0 & 0 & 0 & 0 & 0 & 0 \\
\hline 4. Anorexia sub & 8 & 0 & 0 & 0 & 11 & 0 & 0 & 19 & 1 & 0 & 0 & 0 & 0 & 0 & 0 & 1 \\
\hline 5. Bulimia sub & 18 & 0 & 5 & 1 & 0 & 8 & 1 & 33 & 3 & 0 & 0 & 1 & 0 & 2 & 0 & 6 \\
\hline 6. TPA sub & 3 & 0 & 0 & 0 & 0 & 0 & 1 & 4 & 0 & 0 & 0 & 0 & 0 & 0 & 1 & 1 \\
\hline \multirow[t]{2}{*}{ Total } & 505 & 3 & 31 & 20 & 17 & 25 & 7 & 608 & 349 & 0 & 5 & 4 & 0 & 13 & 1 & 372 \\
\hline & \multicolumn{8}{|c|}{ Mujeres } & \multicolumn{8}{|c|}{ Hombres } \\
\hline Tiempo 2 & \multicolumn{3}{|c|}{ Sin Diag. } & \multicolumn{3}{|c|}{ Cualquier } & \multicolumn{2}{|c|}{ Total } & \multicolumn{3}{|c|}{ Sin Diag. } & \multicolumn{3}{|c|}{ Cualquier } & \multicolumn{2}{|c|}{ Total } \\
\hline \multicolumn{17}{|l|}{ Tiempo 1} \\
\hline Sin diagnóstico & \multicolumn{3}{|c|}{450} & \multicolumn{3}{|c|}{44} & \multicolumn{2}{|c|}{449} & \multicolumn{3}{|c|}{340} & \multicolumn{3}{|c|}{12} & \multicolumn{2}{|c|}{352} \\
\hline Cualquier TCA & \multicolumn{3}{|c|}{55} & \multicolumn{3}{|c|}{59} & \multicolumn{2}{|c|}{114} & \multicolumn{3}{|c|}{9} & \multicolumn{3}{|c|}{11} & \multicolumn{2}{|c|}{20} \\
\hline Total & \multicolumn{3}{|c|}{505} & \multicolumn{3}{|c|}{103} & \multicolumn{2}{|c|}{608} & \multicolumn{3}{|c|}{349} & \multicolumn{3}{|c|}{23} & \multicolumn{2}{|c|}{372} \\
\hline
\end{tabular}

Sub = diagnóstico subumbral; TPA = Trastorno por atracón. 
Tabla 4. Matriz de configuración

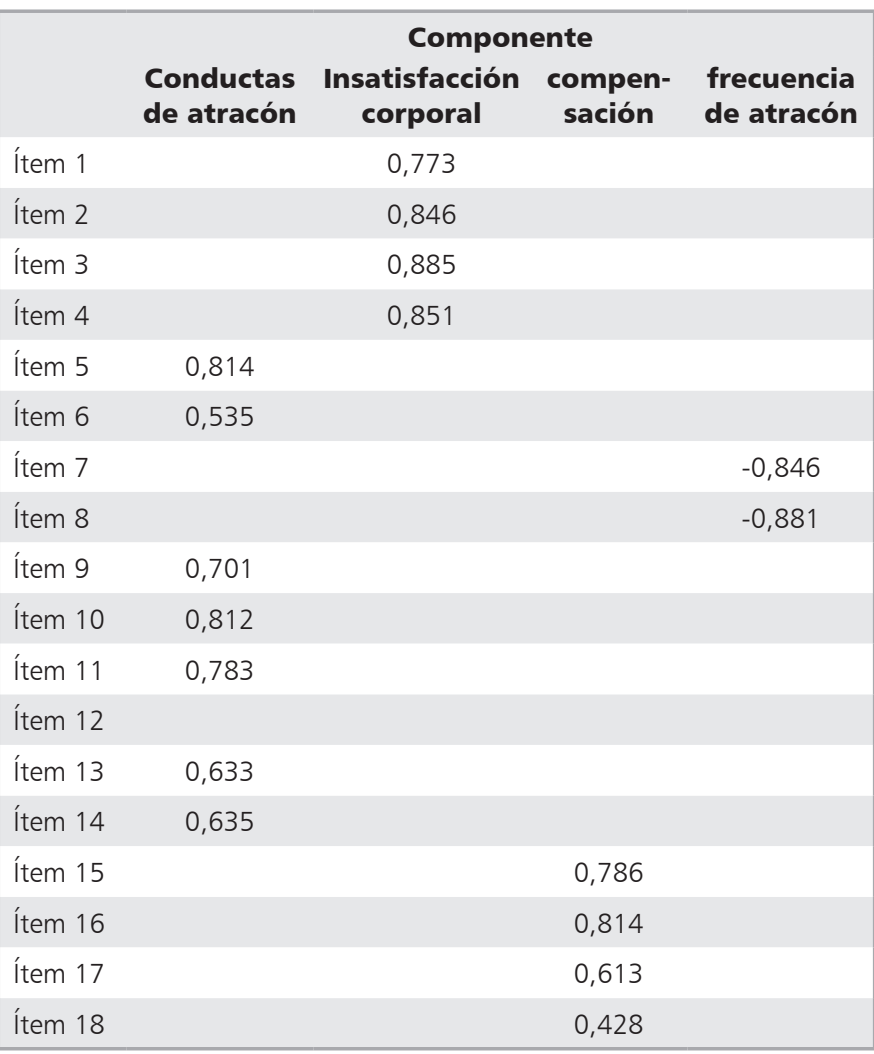

realizó el procedimiento considerando los cuatro factores del EDDS (Tabla 6). Como se observa el compuesto sintomático del EDDS muestra una elevada correlación con Obsesión por la delgadez (EDI-2), Preocupación por la Dieta (RRS), Bulimia (EDI-2) y Conciencia Introceptiva (EDI-2). También se observa una correlación moderada del compuesto sintomático y las fluctuaciones de peso (RRS) Miedo a la Madurez (EDI-2) e Ineficacia (EDI-2). Por otro lado, el factor de Conductas de Atracón obtuvo una alta correlación con Bulimia (EDI2) y moderada con Obsesión por la Delgadez (EDI-2) y Preocupación por Dieta (RSS). El factor de Insatisfacción Corporal obtuvo una alta correlación con Obsesión por la Delgadez (EDI2), Preocupación por la Dieta (RSS) y Conciencia Introceptiva (EDI-2). El factor de Compensación mostró una relación moderada con Obsesión por la Delgadez (EDI-2) y Preocupación por la Dieta (RSS). Finalmente, el factor de Frecuencia de Atracón se asoció moderadamente con Bulimia (EDI-2).

Tabla 5. Correlaciones bivariadas para la muestra total entre el compuesto sintomático del EDDS, el EDI-2 y la RRS

\begin{tabular}{|c|c|c|c|c|c|c|c|c|c|c|}
\hline & $\begin{array}{l}\text { Edds } \\
\text { Com }\end{array}$ & Od & Bul & Insa & Mied & Intro & Inef & Perf & PD & FP \\
\hline EddsCom & 1 & $0,750^{* *}$ & $0,567^{\star *}$ & 0,048 & $0,302^{* *}$ & $0,566^{\star *}$ & $0,365^{* *}$ & $0,261^{* *}$ & $0,682^{* *}$ & $0,480^{* *}$ \\
\hline Od & & 1 & $0,483^{* *}$ & $0,058^{*}$ & $0,344^{* *}$ & $0,562^{* *}$ & $0,329^{* *}$ & $0,268^{* *}$ & $0,763^{* *}$ & $0,432^{* *}$ \\
\hline Bul & & & 1 & 0,045 & $0,340^{* *}$ & $0,568^{* *}$ & $0,379^{* *}$ & $0,293^{* *}$ & $0,362^{* *}$ & $0,367^{\star *}$ \\
\hline Insa & & & & 1 & $0,130^{* *}$ & $0,060^{*}$ & $0,136^{* *}$ & $0,128^{* *}$ & 0,042 & 0,031 \\
\hline Mied & & & & & 1 & $0,460^{* *}$ & $0,390^{* *}$ & $0,348^{* *}$ & $0,235^{* *}$ & $0,170^{* *}$ \\
\hline Intro & & & & & & 1 & $0,521^{* *}$ & $0,354^{* *}$ & $0,432^{* *}$ & $0,288^{* *}$ \\
\hline Inef & & & & & & & 1 & $0,396^{* *}$ & $0,257^{* *}$ & $0,236^{* *}$ \\
\hline Perf & & & & & & & & 1 & $0,202^{* *}$ & $0,183^{* \star}$ \\
\hline PD & & & & & & & & & 1 & $0,441^{\text {** }}$ \\
\hline FP & & & & & & & & & & 1 \\
\hline
\end{tabular}

${ }^{* *} \mathrm{p}<0,01 ;{ }^{*} \mathrm{p}<0,05 ;$ EddsCom = Compuesto sintomático del EDDS; EDI-2: Od = Obsesión por las delgadez; Bul = Bulimia; Insa = Insatisfacción corporal; Mied = Miedo a la madurez; Intro = Conciencia Introceptiva; Inef = Ineficacia; Per = Perfeccionismo; Escala de Restricción: PD = preocupación por la dieta; FP = Fluctuaciones de peso. 
Tabla 6. Correlaciones bivariadas para la muestra total entre 4 factores EDDS, EDI-2 y la RRS

\begin{tabular}{|c|c|c|c|c|c|c|c|c|c|c|c|c|c|}
\hline & CAtr & InsCor & Com & FAtr & Od & Bul & Insa & Mied & Intro & Inef & Perf & PD & FP \\
\hline CAtr & 1 & $0,44^{* *}$ & $0,23^{* *}$ & $0,49^{* *}$ & $0,48^{* *}$ & $0,55^{* *}$ &, 030 & $0,23^{* *}$ & $0,41^{* *}$ & $0,26^{* *}$ & $0,22^{* *}$ & $0,40^{* *}$ & $0,33^{* *}$ \\
\hline InsCor & & 1 & $0,39^{* *}$ & $0,30^{* *}$ & $0,80^{* *}$ & $0,41^{* *}$ & $0,06^{*}$ & $0,26^{* *}$ & $0,50^{* *}$ & $0,31^{* *}$ & $0,23^{* *}$ & $0,74^{* *}$ & $0,48^{* *}$ \\
\hline Com & & & 1 & $0,36^{* *}$ & $0,40^{* *}$ & $0,30^{* *}$ & 0,03 & $0,19^{* *}$ & $0,35^{* *}$ & $0,27^{* *}$ & $0,16^{* *}$ & $0,41^{* *}$ & $0,26^{* *}$ \\
\hline Fatr & & & & 1 & $0,36^{* *}$ & $0,46^{* *}$ & 0,01 & $0,19^{* *}$ & $0,35^{* *}$ & $0,20^{* *}$ & $0,14^{* *}$ & $0,29^{* *}$ & $0,29^{* *}$ \\
\hline Od & & & & & 1 & $0,48^{* *}$ & $0,06^{*}$ & $0,34^{* *}$ & $0,56^{* *}$ & $0,33^{* *}$ & $0,27^{* *}$ & $0,76^{* *}$ & $0,43^{* *}$ \\
\hline Bul & & & & & & 1 & 0,05 & $0,34^{* *}$ & $0,57^{* *}$ & $0,38^{* *}$ & $0,29^{* *}$ & $0,36^{* *}$ & $0,37^{* *}$ \\
\hline Insa & & & & & & & 1 & $0,13^{* *}$ & $0,06^{*}$ & $0,14^{* *}$ & $0,13^{* *}$ & 0,04 & 0,03 \\
\hline Mied & & & & & & & & 1 & $0,46^{* *}$ & $0,39^{* *}$ & $0,35^{* *}$ & $0,24^{* *}$ & $0,17^{\star *}$ \\
\hline Intro & & & & & & & & & 1 & $0,52^{* *}$ & $0,35^{* *}$ & $0,43^{* *}$ & $0,29^{* *}$ \\
\hline Inef & & & & & & & & & & 1 & $0,40^{* *}$ & $0,28^{* *}$ & $0,24^{* *}$ \\
\hline Perf & & & & & & & & & & & 1 & $0,20^{* *}$ & $0,18^{* *}$ \\
\hline PD & & & & & & & & & & & & 1 & $0,44^{* *}$ \\
\hline FP & & & & & & & & & & & & & 1 \\
\hline
\end{tabular}

${ }^{* *} \mathrm{p}<0,01 ;{ }^{*} \mathrm{p}<0,05 ;$ CAtr = Conducta de atracón; InsCor = Insatisfacción corporal; Com = Conducta de compensación; Fatr = Frecuencia de Atracón; EDI-2: Od = Obsesión por las delgadez; Bul = Bulimia; Insa = Insatisfacción corporal; Mied = Miedo a la madurez; Intro = Conciencia Introceptiva; Inef = Ineficacia; Per = Perfeccionismo; Escala de Restricción: PD = preocupación por la dieta; FP = Fluctuaciones de peso.

\section{Estudio 2}

En la Tabla 7 se describe la muestra del estudio 2. Según la entrevista psiquiátrica estructurada 46 participantes padecían de un TCA al momento de la evaluación.

Para comparar el comportamiento del EDDS con el gold standard, se consideró el criterio americano (DSM) y el europeo (ICD). En este caso, se optó por utilizar como criterio la "detección de algún TCA" versus la "no detección”. Se utilizó esta valoración general debido a que el EDDS posee categorías no incluidas en el CIDI. En la Tabla 8 se observa las contingencias entre el EDDS y el diagnóstico psiquiátrico estructurado según los
Tabla 7. Estadísticos descriptivos estudio 2

\begin{tabular}{|lcccc|}
\hline & Sin Diag & TCA & t & p \\
\hline $\mathrm{n}$ & 64 & 46 & & \\
\hline Edad & 19,61 & 20,89 & $-1,778$ & 0,078 \\
IMC & 23,40 & 21,78 & 1,819 & 0,072 \\
\hline EddsCom & 22,98 & 45,27 & $-7,571$ & $0,000 * *$ \\
\hline
\end{tabular}

${ }^{* *} p<0,01 ;{ }^{*} p<0,05 ;$ Diag = Diagnóstico; TCA $=$ Trastorno de la conducta alimentaria; IMC = Índice de Masa Corporal; EddsCom = Compuesto sintomático del EDDS; CAtr = Conducta de atracón; InsCor = Insatisfacción corporal; $\mathrm{Com}=$ Conducta de compensación; Fatr $=$ Frecuencia de Atracón.

Tabla 8. Tabla de contingencias entre EDDS y CIDI (criterios ICD y DSM)

\begin{tabular}{|lcccccc|}
\hline CIDI & Sin Diag. & $\begin{array}{c}\text { Criterio ICD } \\
\text { Cualquier }\end{array}$ & Total & Sin Diag. & $\begin{array}{c}\text { Criterio DSM } \\
\text { Cualquier }\end{array}$ & Total \\
\hline Sin diagnóstico & 47 & 9 & 56 & 50 & 6 & 56 \\
Cualquier TCA & 16 & 37 & 53 & 14 & 39 & 53 \\
\hline Total & 63 & 46 & 109 & 64 & 46 & 109 \\
\hline
\end{tabular}

El Kappa para el acuerdo entre el EDDS y el CIDI-ICD fue de 0,54 (moderado), mientras que para el EDDS y CIDI-DSM fue de 0,63 (moderado-alto) 
criterios de diagnósticos de enfermedades psiquiátricas (desórdenes alimentarios) para el ICD y el DSM.

\section{Discusión}

Los datos de los estudios realizados permiten confirmar las cinco hipótesis asociadas. Tal como se señalará a continuación, cada afirmación resultó respaldada completa o parcialmente por los resultados obtenidos:

El EDDS es un instrumento psicométrico confiable con un alpha de Cronbach de 0,84 para la muestra total, es decir, con un muy buen indicador de confiabilidad de los ítems, con niveles de fiabilidad test/re-test moderados. Efectivamente, al considerar tanto las 7 categorías diagnósticas como la detección de un cualquier diagnóstico se observó un Kappa moderado, acorde a estudios internacionales previos ${ }^{23}$, que reportan rangos de Kappa entre 0,40 y 0,48.

La versión en español del EDDS presentó una estructura factorial similar a la versión original, en el caso de las mujeres. Los factores fueron "insatisfacción corporal", "conductas de atracón", "frecuencia de atracón" y de "frecuencia de conductas compensatorias" 23 , develando una excelente adecuación de la estructura factorial. No obstante, en el caso de los hombres se obtuvo una estructura factorial diferente, incluyendo dos de los factores previamente descritos más otros cuatro. Esta diferencia requiere ser analizada por estudios futuros, pero condiciona el uso del EDDS para investigaciones que pretenden utilizar explícitamente los cuatro factores. En tales casos la recomendación es hacer uso del EDDS sólo en población de mujeres. Así, la hipótesis de la estructura factorial se confirmó para las mujeres y parcialmente para hombres.

El diagnóstico de TCA según el EDDS se asoció a niveles elevados de sintomatología alimentaria evaluada con el EDI-2. Se observó un grado de validez convergente apropiada según las correlaciones entre los factores del EDDS y las puntuaciones del EDI-2 y la RRS.

Finalmente, el diagnóstico de TCA obtenido con el EDDS es concordante con el obtenido mediante el gold standard CIDI. Esta hipótesis se cumplió moderadamente para el criterio diagnóstico europeo $(I C D$, Kappa $=0,54)$ y satisfactoriamente para el diagnóstico americano (DSM, Kappa $=0,63$ ). Los datos develados por el estudio indican que el EDDS tiene un moderado-bueno nivel de identificación de casos de TCA. De este modo, en el contexto clínico puede ser una buena herramienta de apoyo para un diagnóstico económico de sospechas de patología alimentaria.



Figura 1. Porcentaje de diagnósticos según el EDDS en mujeres en relación a muestra total $(n=1.964)$. Nota al pie: $E D D S=$ Escala de Diagnóstico de Desórdenes Alimentarios; An_sub=Anorexia sub-umbral; Bul sub=Bulimia sub-umbral; At sub=Trastorno por Atracón sub-umbral.

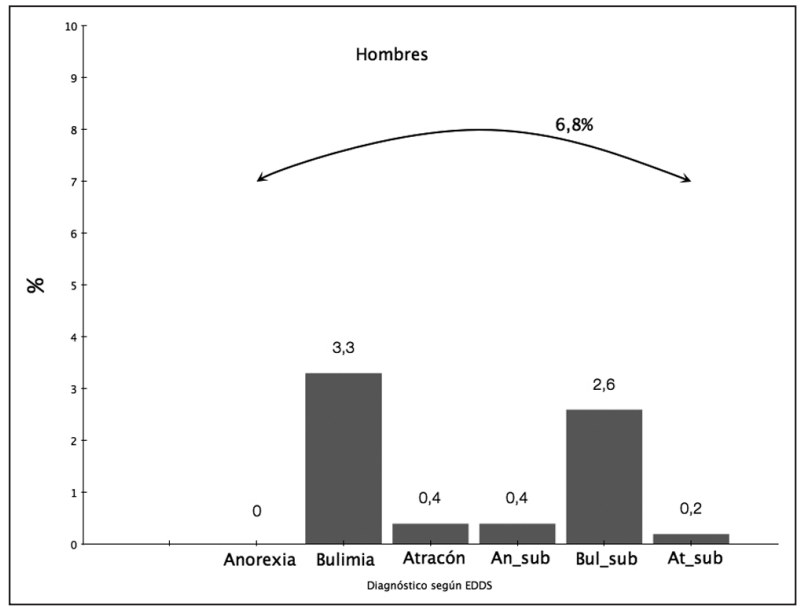

Figura 2. Porcentaje de diagnósticos según el EDDS en hombres en relación a muestra total $(n=1.964)$. Nota al pie: $E D D S=$ Escala de Diagnóstico de Desórdenes Alimentarios; An sub=Anorexia sub-umbral; Bul_sub=Bulimia sub-umbral; At_sub=Trastorno por Atracón sub-umbral. 
Por otra parte, los datos obtenidos con el uso del EDDS en escolares y estudiantes universitarios demuestran que los TCA son un problema relevante de salud. En efecto, tal como se observa en la Figura 1 y 2, el 17,8\% de las mujeres encuestadas presentaron problemas en esta esfera de la conducta. La bulimia nerviosa alcanza una presencia de $6,1 \%$ del total de evaluados. Además, el 6,8\% de los hombres reportaron algún tipo de alteración alimentaria, incluyendo $3,3 \%$ de presencia de bulimia. Esta información debe considerarse para (a) el fortalecimiento de investigación básica y aplicada en el área y (b) el establecimiento de políticas explícitas para el abordaje de la problemática, tanto en los niveles epidemiológico, preventivo y de intervención.

En conclusión, la versión en español del EDDS mostró un comportamiento psicométrico satisfactorio y una buena capacidad para detectar casos de TCA según el criterio DSM. Así, el estudio presente puede considerarse como un paso necesario para la generación de las condiciones favorables para el abordaje clínico (desde el nivel primario en adelante), epidemiológico y científico de los TCA en Chile. Indudablemente, no es el único esfuerzo necesario, pero sí uno fundamental para sustentar gran parte de las acciones relativas al abordaje de los TCA a nivel nacional. El uso del EDDS puede recomendarse para la investigación científica, ya sea en (a) la identificación de casos de TCA como para (b) la detección de variaciones en presencia de problemáticas asociadas a la patología alimentaria. En este último caso, se recomienda sólo el uso del EDDS en mujeres. Por otra parte, en el ámbito clínico, los resultados del estudio apoyan la recomendación de su uso como herramienta diagnóstica complementaria y de apoyo al diagnóstico clínico.

Agradecimientos: Los autores agradecen a Yessenia Basterrechea, Mario Beroiza, Stefanella Costa, Bárbara Graf, María Eugenia Meneses, Humberto Pizarro, Camila Román, Mónica Sanhueza, Bettina Santamaría y Tatiana Vega por su colaboración en la recolección y/o ingreso de datos.

\section{Referencias}

1. Behar R, Arriagada MI, Casanova D. [Eating and affective disorders: A comparative study]. Rev Med Chile 2005; 133 (12): 1407-14.
2. Behar R. [Eating disorders in adolescents: Epidemiological and clinical aspects]. Rev Med Chile 1998; 126 (9): 1085-92.

3. Cordella P. Proposición de un modelo para comprender la configuración anoréctica. Rev Chil Pediatr 2002;76 (6): 566-75.

4. Behar R. Trastornos de la conducta alimentaria: Clínica y epidemiología. En: Behar R, Figueroa G, editores. Trastornos de la conducta alimentaria. Santiago, Chile: Editorial Mediterráneo; 2010. p. 121-47.

5. Behar R. Quince años de investigación en trastornos de la conducta alimentaria. Rev Chil Neuropsiquiat 2010; 48 (2): 135-46.

6. Cordella P. Anorexia, bulimia y obesidad: Experiencia $y$ reflexión con pacientes y familias. Santiago de Chile: Ediciones UC; 2010.

7. Cassin SE, von Ranson KM. Personality and eating disorders: a decade in review. Clin Psychol Rev. 2005; 25 (7): 895-916.

8. Colles S, Dixon J, O’Brien P. Binge eating disorder and the night eating syndrome: Prevalence in subgroups of the Australian population, and association with body weight and psychopathology. Obes Surg 2006; 16 (8): 983-4.

9. Gluck ME. Stress response and binge eating disorder. Appetite 2006; 46 (1): 26-30.

10. Arriagada M, Behar R. Trastornos de la conducta alimentaria no especificados. En: Behar R, Figueroa G, editores. Trastornos de la conducta alimentaria. Santiago de Chile: Mediterráneo; 2010. p. 148-66.

11. Alves E, de Vasconcelos FDG, Calvo MCM, das Neves J. Prevalence of symptoms of anorexia nervosa and dissatisfaction with body image among female adolescents in Florianopolis, Santa Catarina State, Brazil. Cad Saude Publica 2008; 24 (3): 503-12.

12. Hoek HW. Incidence, prevalence and mortality of anorexia nervosa and other eating disorders. Curr Opin Psychiatry 2006; 19 (4): 389-94.

13. Sodersten P, Bergh C, Bjoernstroem M. Prevalence and recovery from anorexia nervosa. Am J Psych 2008; 165 (2): 264-5.

14. Correa M, Zubarew G, Silva P, Romero M. Prevalencia de riesgo de trastornos alimentarios en adolescentes mujeres escolares de la Región Metropolitana. Rev Chil Pediatr 2006; 77 (2): 153-60.

15. Johnson JG, Spitzer RL, Williams JB. Health problems, impairment and illnesses associated with bulimia nervosa and binge eating disorder among primary care and obstetric gynaecology patients. Psychol Med 2001; 31 (8): 1455-66.

16. Behar R. Trastornos de la conducta alimentaria no especificados, síndromes parciales y cuadros subclínicos: Una 
Validación de la versión en español del Eating Disorder Diagnostic Scale - J. R. Silva et al

alerta para la atención primaria. Rev Med Chile 2008; 136 (12): 1589-98.

17. Turnbull S, Ward A, Treasure J, Jick H, Derby L. The demand for eating disorder care. An epidemiological study using the general practice research database. $\mathrm{Br} \mathrm{J}$ Psychiatry. 1996; 169 (6): 705-12.

18. Garner DM, Garfinkel PE. The eating attitudes test: An index of the symptoms of anorexia nervosa. Psychol Med 1979; 9: 273-9.

19. Garner DM. Eating disorder inventory-2 Manual. Odessa, FL: Psychological Assessment Resources; 1991.

20. Stice E, Telch CF, Rizvi SL. Development and validation of the Eating Disorder Diagnostic Scale: a brief selfreport measure of anorexia, bulimia, and binge-eating disorder. Psychol Assess 2000; 12 (2): 123-31.

21. Stice E, Fisher M, Martínez E. Eating disorder diagnostic scale: additional evidence of reliability and validity. Psychol Assess 2004; 16 (1): 60-71.

22. Chen H, Jackson T. Prevalence and sociodemographic correlates of eating disorder endorsements among adolescents and young adults from China. Eur Eat Disord Rev 2008; 16 (5): 375-85.

23. Lee SW, Stewart SM, Striegel-Moore RH, Lee S, Ho SY, Lee PW, et al. Validation of the eating disorder diagnostic scale for use with Hong Kong adolescents. Int J Eat Disord 2007; 40 (6): 569-74.

24. Tabachnick BG, Fidell LS. Using multivariate statistics. Boston: Allyn \& Brown; 2001.

25. Urzúa A, Castro S, Lillo A, Leal C. Evaluación de los trastornos alimentarios: propiedades psicométricas del test edi-2 en adolescentes escolarizados(as) de 13 a 18 años. Rev Chil Nutr 2009; 36 (4): 1063-73.

26. Heatherton TF, Herman CP, Polivy J, King GA, McGree
ST. The (mis)measurement of restraint: an analysis of conceptual and psychometric issues. J Abnorm Psychol 1988; 97 (1): 19-28.

27. Herman CP, Polivy J. Restrained Eating. En: Stunkard A, editor. Obesity. Philadelphia: Saunders; 1980. p. 208-25.

28. Silva JR. Sobrealimentación inducida por la ansiedad, Parte I: Evidencia conductual, afectiva, metabólica y endocrina. Ter Psicol 2007; 25 (2): 141-54.

29. Silva JR. Sobrealimentación inducida por la ansiedad, Parte II: Un marco de referencia neurocientífico para el desarrollo de técnicas psicoterapéuticas. Ter Psicol 2008; 3 (1): 99-115.

30. Silva JR. Restricción alimenticia y sobrealimentación: Un modelo de la neurociencia afectiva. Rev Med Chile 2008; 136 (10): 1336-42.

31. Silva JR, Urzúa A. Propiedades psicométricas de la versión en español de la escala revisada de restricción alimentaria en una muestra de adolescentes. Univ Psychol 2010; 9 (2), 521-30.

32. Silva JR. Consistencia interna y validez factorial de la versión en español de la escala revisada de restricción alimentaria. Rev Chil Nutr 2010; 37 (1): 41-9.

33. World Health Orgzanization. The Composite International Diagnostic Interview (CIDI). Geneve, Switzerland: World Health Organization, 1997.

34. American Psychiatric Association. Diagnostic and statistical manual of mental disorders. Text Revision. 4th ed. Washington (DC): American Psychiatric Association; 2000.

35. World Health Orzanization. The ICD-10 classification of mental and behavioural disorders: Clinical descriptions and diagnostic guidelines. Geneve: World Health Orgzanization; 1992. 